ISBN: 978-1-948012-12-6

\title{
PULSE EDDY CURRENT NONDESTRUCTIVE TESTING
}

\author{
Cai Jiabao, Wang Haibo*
}

Power Engineering and Engineering Thermophysics, Jilin Institute of Chemical technology, Chengde Street, Jilin City, Jilin, China. *Corresponding Author Email: wanghaibo197426@163.com

This is an open access article distributed under the Creative Commons Attribution License, which permits unrestricted use, distribution, and reproduction in any medium, provided the original work is properly cited.

\section{ARTICLE DETAILS}

\section{Article History:}

Received 26 June 2018 Accepted 2 July 2018 Available online 1 August 2018

\section{ABSTRACT}

Pulse eddy current nondestructive testing technology is part of eddy current testing technology, The wide-spectrum excitation method makes this technology widely used in the aerospace field, railway transportation and pipelines, pressure vessels and other fields. This review describes pulse eddy current testing techniques in material defects, Research progress in stress detection and thermal imaging technology. Summarized and analyzed the development trend of pulse eddy current inspection technology.

\section{KEYWORDS}

Pulse vortex, Defect, Stress, Thermal imaging.

\section{INTRODUCTION}

As a very promising non-destructive testing technology, Pulse eddy current non-destructive test whose pulse contains a wide spectrum, the sensed voltage signal contains a lot of material status information [1]. The NDT technique uses matrix pulses as the input signal. Generate a magnetic field through the excited coil, the magnetic field produces induced eddy currents in the measured conductor parts [2]. At the same time, this induced eddy current is affected by the magnetic conductivity and electrical conductivity of the parts, the influence factors of permeability and conductivity are related to the composition, strength, stress strain and defects of the tested part [3]. The conductivity and permeability of the alternating magnetic field of the eddy current can be detected by the detection coil, and finally the non-destructive detection of the tested part is obtained. Therefore, pulsed eddy current non-destructive testing technology is widely used in aerospace fields, railway transportation, pipelines, pressure vessels and other fields. The pulse eddy current nondestructive testing method is a part of the eddy current testing technology. It can quantitatively detect the depth of defects on the surface and near surface of conductor materials. For the metal parts with internal defects, the pulse eddy current testing method is a very effective defect detection technology [4]

\section{ADVANTAGES OF PULSED EDDY CURRENT TESTING} TECHNOLOGY

\section{$2.1 \quad$ Defect Detection}

Pulse eddy current testing technology is a unique high-speed large-size detection technology. Compared with X-ray inspection technology, pulsed eddy current testing technology has no radiation damage to itself. It not only can work in harsh environments, but also has low equipment cost. The required operating level is not high; in comparison with ultrasonic testing, it has the advantage of not using a couplant; it is suitable for any conductive medium with respect to magnetic particle detection technology; in comparison with liquid penetrant testing It can not only detect the surface defect of the object but also detect the internal defect [5].

\section{$2.2 \quad$ Stress detection}

Compared with other non-destructive testing methods, pulse eddy current stress detection method is more effective. Compared with X-ray detection technology, pulsed eddy current is less affected by grain size, while X-ray can only measure surface macro and micro stress or residual stress [6]. Not only is the equipment expensive and requires a relatively high level of detection technology. Compared with the diffraction detection technology, ultrasonic detection technology has a deeper detection depth, but it also has the disadvantage of having to use a couplant, and it is difficult to detect small and complex objects and surfaces with uneven materials. Other measurement methods such as synchrotron diffraction and neutron diffraction have high resolving power [2]. However, the test equipment is very scarce and can only be possessed by a few laboratories in Europe and the United States. The material's sub-resistance effect has great advantages in the measurement of residual stress in pulsed eddy current testing methods. Researchers at home and abroad have done a lot of experimental research to prove the eddy current non-destructive testing on the relationship between the conductivity of the material and stress. Technology is effective in detecting the stress of magnetic and nonmagnetic materials [7].

\subsection{Pulsed eddy current thermal imaging}

Pulsed eddy current non-destructive testing technology is complementary to other non-destructive testing technologies [8]. With the continuous progress of signal processing technology, pulsed eddy current nondestructive testing methods have been widely used in the detection of parts defects in the aerospace industry. Pulsed eddy currents are used to heat the outer surface of the metal, and the eddy currents generated by the alternating pulsed magnetic field can rapidly heat the metal surface [9]. Then the infrared thermal imaging method is used to image the defect as a pulsed eddy current thermal imaging method. Its imaging technology can quickly detect large areas of parts in a short period of time and is suitable for almost all material inspections.

\section{PULSE EDDY CURRENT NONDESTRUCTIVE TESTING AT HOME AND ABROAD}

\subsection{The main research status in foreign countries}

After the 1970s, with the continuous development of science and technology, electronic technology and computer technology have also achieved leap-forward progress. In the field of eddy current nondestructive testing technology, the technical performance of its equipment 
has been greatly improved. The superiority of detecting metal surface defects and other advantages is further highlighted.

Among them, a researcher at the State University of Iowa in the United States found in experiments that the peak value of the pulsed eddy current detection signal has a certain relationship with the degree of metal corrosion [10]. According to the influence of the zero-crossing time on the corrosion degree, the test result is provided. Based on the results of its research, the instrument for corrosion detection was designed specifically for the detection of aircraft fuselage [8].

Prof. Yun Tian from Newcastle University in the UK used Ansys software to find out that the conductivity of the metal part affected the peak value of the pulsed eddy current differential signal by performing the finite element model simulation. The conductivity is then the same as the stress of the material. The deformation is related to the method of the pulse eddy current to evaluate the stress [11]

Smith Robert A and others in the United Kingdom have successfully developed a pulsed eddy current nondestructive testing instrument. For the first time, they used a Hall sensor instead of a wound coil as a detection component for pulsed eddy current testing [4]. Surprisingly, it is found that the Hall sensor has a better low-frequency response and is more suitable for deep metal defect detection.

Canada's BALepine found that under certain conditions, the amount of metal corrosion does not change. At this time, no matter how the sensor's measurement distance changes, the pulse signal will appear together as a crossover point, and we call it the "lift off intersection". Using this intersection can eliminate interference and improve detection accuracy [12].

\subsection{Main Research Status in China}

Researchers in China began research on pulsed eddy current nondestructive testing technology in the 1960s. Although they started late, they have made rapid progress. So far, Chinese universities and research institutes have carried out relevant research on eddy current testing technology and obtained.

In the 1970s, Prof. Tan Zugen and Prof. Chen Shouchuan from Zhejiang University summarized the directions of non-destructive testing for eddy currents in various countries around the world, and then divided the eddy current nondestructive testing circuit into three categories: 1 . Frequencymodulated amplitude modulation circuit 2 . Constant-frequency amplitude modulation circuit 3 . Frequency-modulated circuit. It laid the foundation for the development of eddy current testing equipment.

Professor Zheng Feijun of Dalian University of Technology and Professor Luo Tiyun of the National University of Defense Technology processed eddy current signals using principal component analysis. They applied this analysis method to lifting effect, measuring metal thickness and processing corrosion edge signals, and achieved good results [13].

Professors Fan Mengpai and Cao Binghua of China University of Mining and Technology have established a mathematical model for the application of pulsed eddy current differential probe output signals in flat panel metal detection, and established an analytical model for exciting the probe impedance changes under harmonic excitation [14]. At the same time, the knowledge of Laplace transform is applied to the establishment of a time-domain analytical model of the transient eddy current field of pulse eddy currents. Moreover, the theoretical system of pulse eddy currents has been improved as a whole [4].

\section{DEVELOPMENT TRENDS OF PULSED EDDY CURRENT TESTING}

With the continuous development of the market globalization, eddy current testing technology has also experienced the development phase from single-frequency vortex to multi-frequency vortex to the current pulse eddy current. Due to the constant efforts of researchers in the world, eddy current non-destructive testing will be more in-depth research and greater progress will be made. In general, pulse eddy current testing technology will develop in the following areas:

\subsection{Far-field eddy current nondestructive testing}

The detection of large-caliber metal tubes can generally use far-field eddy current detection technology [15]. The probes adopt internal-pass probes whose composition includes detection coils and excitation coils. The excitation coil can receive a low-frequency current signal, and the excited eddy current signal passes through the wall of the pipe to the outside of the pipe and is then received by the detection coil. This technology not only can detect the defect condition of the metal pipe, but also can measure the thickness of the pipe [10]. As the technology is continuously known by people, the importance of far field eddy current testing technology has gradually been recognized by the public.

\subsection{Magneto-optical eddy current testing technology}

Similar to far-field eddy current non-destructive testing, magneto-optical eddy current testing technology also uses excitation coils to generate magnetic fields on backside materials. Researchers use Tu Fuming's magnetic field sensor consisting of thin slices of stone-ferrite material to measure the surface of the object under test. The pulsed eddy currents and the induced magnetic field generated by the pulsed eddy currents are reflected by different out-of-plane-angle signals, and then the chargedevice CCD is used to receive the magneto-optical signals, which are displayed on the detector by the detection device [7].

\subsection{Eddy current array detection technology}

The eddy current array detection technology has been used for more than 10 years after the development process $[16,17]$. The technology uses arrayed arrays of multiple sensors to work together. Because it is subject to stringent data analysis, calculation, and processing capabilities, it has been continuously developed. Slowly, compared with other eddy current testing techniques, eddy current array detection technology has the advantages of rapid detection and higher sensitivity.

\section{CONCLUSION}

Compared with other non-destructive testing methods, pulsed eddy current non-destructive testing technology has distinct advantages in metal defects, stress, and thermal imaging detection. Based on the above research progress at home and abroad, we need to further study the following aspects:

1. In addition to the cylindrical probe or rectangular probe analysis method, it is necessary to further explore the structure theory of other shape probes.

2. The current difficulty in pulse non-destructive testing is the extraction, classification, identification and defect quantification of pulse eddy current defect signal characteristics.

3. It is necessary to further study the influence of factors such as chemical composition, microstructure, hardness, surface roughness, and temperature of the conductive material, and to improve the measurement accuracy of the corresponding force of the pulsed eddy current technique.

4. The detection depth of the pulsed eddy current thermal imaging technique with the excitation frequency up to $149 \mathrm{kHz}$ is not ideal, and the detection depth of the pulsed eddy current thermal imager is still the focus of current research.

5. Based on the integration of other electromagnetic non-destructive testing techniques and pulsed eddy current testing technology, the stress, structural changes and complex defects detection of materials are currently the research direction of this technology.

\section{REFERENCES}

[1] Zhao, Y., Xie, S.J. 2018. Frequency-selective pulse eddy current detection method for known depth range defects. China Mechanical Engineering, (06), 639-644. 
[2] Wu, X.J. 2016 A review of pulsed eddy current non-destructive testing technology. Journal of Instrumentation, 37 (08), 1698-1712.

[3] Uzal, E., Moulder, J.C., Mitra, S. 1993. Impedance of coils over layered metals with con-tinuously variable conductivity and permeability; theory and experiment. Journal of Applied Physics, 74 (3), 2076-2089.

[4] Duan, X.S. 2014. Research on Pulsed Eddy Current Nondestructive Testing Technology. Tianjin University.

[5] Li, Y.S. 2014. Study on Frequency Domain Feature Extraction and Defect Characterization of Pulsed Eddy Current Detection. Xiamen University.

[6] Pan, H. 2015. Non-destructive testing of ferromagnetic components using pulse eddy currents. Jiangsu University of Technology.

[7] Blodgett, M.P., Nagy, P.B. 2004. Eddy current assessment of nearsurface residual stress in shot-peenednickel-basesuperalloys. Journal of Nondestructive Evaluation, 23 (3), 107-123.

[8] Wang, S.S., Yan, Z. 2015. Pulse eddy current non-destructive testing technology and its application in defect detection of aerospace materials. Spacecraft Environment Engineering, 32 (03), 307-312.

[9] Xie, J., Xu, C.H., Zhou, Y.W. 2015. Design of non-destructive testing of pulsed eddy current infrared thermal image. Laboratory Research and Exploration, 34 (09), 58-61.

[10] Duan, C.G. 2013. Pipeline Detection Method Based on Pulsed Eddy
Current. Shenyang University of Technology.

[11] Luquire, J.W., Deeds, W.E., Dodd, C.V. 1970. Alternating current distribution between planar conductors. Journal of Applied Physics, 41 (10), 3983-3991.

[12] Brunei, B., Jayet, Y., Baboon, J.C. 1995. Pulsed eddy current application to the detection of deep cracks. J. Materials Evaluation, 53 (11), 12961300 .

[13] Cao, H.X., Wang, C., Yang, B.F. 2011. Study on lift-off effect of pulse eddy current non-destructive testing. Journal of Air Force Engineering University (Natural Science Edition), 12 (03), 45-49.

[14] Zhao, H.L., Eng, W.Y., Huang, X. 2017. Parametric Model Analysis of Eddy Current Nondestructive Testing Based on System Discrimination. Electric Automation, 39 (06), 106-108.

[15] Hou, C.M. 2017. Research on theory and technology of nondestructive testing of submarine pipelines based on pulsed vortex. Dalian University of Technology.

[16] De'nan, F., Nazri, F.M., Hashim, N.S. 2017. Finite Element Analysis on Lateral Torsional Buckling Behaviour Oi I-Beam with Web Opening. Engineering Heritage Journal, 1 (2), 19-22.

[17] Shaikh, M.M., AlSuhaimi, A.O., Hanafiah, M.M., Ashraf, M.A., Fantoukh, A., AlHarbi, E. 2017. Leachable Volatile Organic Compounds from Polyethylene Plumbing Plastic Pipes: a case study of Medina Al Munawarah, Saudi Arabia. Acta Chemica Malaysia, 1 (1), 01-03. 